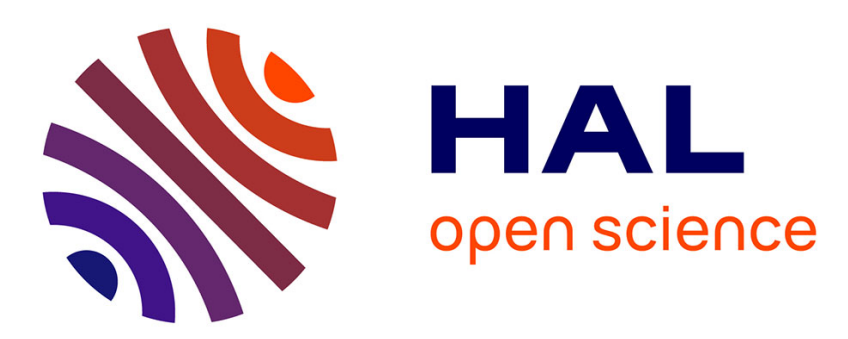

\title{
Focusing Wave Group Propagating in Finite Depth in the Presence of Surface Current and Vorticity
}

\author{
Julien Touboul, Christian Kharif
}

\section{To cite this version:}

Julien Touboul, Christian Kharif. Focusing Wave Group Propagating in Finite Depth in the Presence of Surface Current and Vorticity. Nonlinear Waves and Pattern Dynamics, Springer International Publishing, pp.77-90, 2018, 10.1007/978-3-319-78193-8_4 . hal-02046506

\section{HAL Id: hal-02046506 https://hal.science/hal-02046506}

Submitted on 22 Feb 2019

HAL is a multi-disciplinary open access archive for the deposit and dissemination of scientific research documents, whether they are published or not. The documents may come from teaching and research institutions in France or abroad, or from public or private research centers.
L'archive ouverte pluridisciplinaire HAL, est destinée au dépôt et à la diffusion de documents scientifiques de niveau recherche, publiés ou non, émanant des établissements d'enseignement et de recherche français ou étrangers, des laboratoires publics ou privés. 


\title{
Focusing Wave Group Propagating in Finite Depth in the Presence of Surface Current and Vorticity
}

\author{
Julien Touboul and Christian Kharif
}

\begin{abstract}
The kinematics of two-dimensional focusing wave trains on a shearing flow in water of finite depth are investigated analytically. In the absence of waves, the vorticity due to the vertical gradient of the horizontal current velocity is assumed constant. A linear kinematic model based on the spatio-temporal evolution of the frequency is derived predicting the focusing distance and time of a chirped wave packet in the presence of constant vorticity, and surface current. Based on this model, the kinematic behavior of the transient wave packet is analyzed, and described in terms of spreading of the focusing point into a wider area. The effects of bathymetry, vorticity, and surface current are analyzed and discussed. Two main results are obtained: (i) the combined effects of surface current and vorticity, in deep water, are nontrivial, highly depending on the presence of surface current (ii) the effects of bathymetry, in the presence of shear, are also counterintuitive in the presence of vorticity, since significant effects can be observed when considering high values of the depth parameter.
\end{abstract}

\section{Introduction}

Freak, rogue, or giant waves are extreme events, localized in time and space. Most of the time, they are defined by a wave height briefly exceeding some statistical properties of the wave field, such as twice the significant wave height. They are characterized by their unpredictability, which explains that they are known as "waves from nowhere". They are responsible for an important number of large damages, caused to ships or offshore rigs, which explains they have focused the attention of the scientific community for the last 30 years.

J. Touboul ( $\otimes)$

CNRS / INSU, IRD, MIO, Université de Toulon, Aix-Marseille Université,

83957 UM, 110, La Garde, France

e-mail: julien.touboul@mio.osupytheas.fr

C. Kharif

CNRS, Centrale Marseille, IRPHE, Aix-Marseille Université, Marseille, France

e-mail: kharif@irphe.univ-mrs.fr 
Formation of these waves might be explained through various mechanisms, such as spatio-temporal focusing Kharif et al. [15], Johannessen and Swan [11], Brown [4], Brown and Jensen [5], nonlinear or modulational instability Benjamin and Feir [2], Dyachenko and Zakharov [9], envelope soliton and breather interactions Clamond and Grue [6]. These mechanisms have been reviewed by Kharif and Pelinovsky [14] and by Dysthe [10].

Wave-current interaction contributes also in the freak wave formation and historically; this mechanism was the first to explain the origin of freak waves Lavrenov [20], White and Fornberg [35]. This is due to an important modification of water waves kinematics in the presence of currents reviewed extensively in Peregrine [23] and Jonsson [13].

Since all these mechanisms can exist simultaneously at sea, it is interesting to analyze their robustness when considered together. This, for instance, was achieved for studying the influence of sheared currents on modulational instability in Johnson [12]. The influence of wind on dispersive focusing was studied in Touboul et al. [27], and in Kharif et al. [16], while the evolution of modulation instability under wind action was studied in [17, 30, 31].

Such a question can be asked about the dispersive focusing under the action of currents. This was initially addressed by Touboul et al. [28], who investigated the modification of the dispersive focusing mechanism for water waves propagating in deep water in the presence of uniform currents. But generally, the characteristic length scales of wind waves and swells are much smaller than the spatial scales of horizontal variations in oceanic currents. On the opposite, there are many circumstances in which this claim cannot be applied to current velocity variations in vertical direction. Consequently, the vorticity due to the vertical gradient of the horizontal current velocity which may have an important effect on the dynamics of ocean surface waves cannot be ignored. Various studies of water waves propagating in such conditions can be found in the literature. Among them, one can cite Tsao [33], Dalrymple [8], Brevik [3], Simmen [24], Simmen and Saffman [25] Teles da Silva and Peregrine [26], Kishida and Sobey [19], Pak and Chow [22], Constantin [7], etc. Thus, Touboul and Kharif [32] extended their previous study [28] to this more realistic case of water waves propagating in the presence of vorticity.

Besides, such vortical flows are observed in coastal areas [1, 29]. As pointed out by Professor Ezersky, the effect of bathymetry on dispersive properties should be taken into account. This study was realized experimentally and numerically for water waves propagating in waters of finite depth, in the presence of uniform currents in Merkoune et al. [21].

The purpose of this work is to analyze both the effects of surface current and vorticity for rogue waves propagating in waters of finite depth, extending results presented in [21] to take vorticity into account. To achieve this goal, attention is focused on the kinematic behavior of a focusing wave group. The components should merge at a given point in both time and space, producing a rogue wave. The transformation of this point into a wider area, under the combined effects of surface current, vorticity, and depth is investigated here. The chapter is organized as follows: in Sect. 2, the kinematic model is formulated, focusing on the computation 
of the coordinates of the focusing point without and with current and vorticity, in finite depth. In Sect. 3, the results are discussed in detail, and briefly summarized in Sect. 4, describing the main conclusions of the work.

\section{The Kinematic Model}

Since water waves are known to be dispersive, short waves propagating in front of longer ones will be overtaken, and a large amplitude wave can occur at a fixed point. A linear approach of the problem would lead to consider sea surface as a superposition of linear waves of frequencies $\omega(x, t)$. The following nonlinear hyperbolic equation governs the spatiotemporal evolution of these components, as pointed out by Brown [2] and Whitham [34],

$$
\frac{\partial \omega}{\partial t}+C_{g}(\omega) \frac{\partial \omega}{\partial x}=0,
$$

where $C_{g}$ is the group velocity, defined as $C_{g}=\partial \omega / \partial k$. The boundary value problem for this equation can be solved using the method of characteristics. Its solution is

$$
\omega(x, t)=\omega_{0}(\tau), \text { on } t=\tau+x / C_{g}(\omega),
$$

where $\omega_{0}$ corresponds to the temporal frequency distribution of the wave train at $x=0$. By differentiating the frequency, it comes

$$
\frac{\partial \omega}{\partial t}=\frac{\frac{\partial \omega_{0}}{\partial \tau}}{1-\frac{x}{C_{g}^{2}} \frac{\partial C_{g}}{\partial \omega} \frac{d \omega_{0}}{d \tau}}
$$

The latter equation can be solved once the dispersion equation is known, and various examples of solutions can be found in the literature. In the classical formulation of water propagating in deep water, for instance, this dispersion equation reads

$$
\omega^{2}=g k
$$

where $k$ stands for the wavenumber, and $g$ is the acceleration due to gravity. We can now obtain an expression of the group velocity given by $C_{g}=g /(2 \omega)$. Equation (3) might thus be rewritten

$$
\frac{\partial \omega}{\partial t}=\frac{\frac{\partial \omega_{0}}{\partial \tau}}{1-\frac{2 x}{\mathrm{~g}} \frac{d \omega_{0}}{d \tau}},
$$

and one can notice that the case $d \omega_{0} / d \tau<0$, which corresponds to the case of short waves emitted before longer waves, leads to a singularity. This singularity 
corresponds to the focusing of several waves at $t=T_{f}$ and $x=X_{f}$. Given the expression of the group velocity in the case at hand, we can find out the frequency to impose to a wave maker located at $x=0$, and for $0<t<T$, given by

$$
\omega(0, t)=\frac{g}{2} \frac{T_{f}-t}{X_{f}}
$$

This frequency modulation, varying linearly from $\omega_{\min }$ to $\omega_{\max }$, provides the optimal focusing of the linear wave packets in still water of infinite depth, and is very often applied in the laboratory conditions. Such a wave train will involve components which will propagate and all merge at the same place $X_{f}$, and at the same time $T_{f}$. Coordinates of the focusing point in the $(x-t)$ plane would thus be given by

$$
X_{f}=\frac{g T}{2} \frac{1}{\omega_{\max }-\omega_{\min }}, \text { and } T_{f}=\frac{2 \omega_{\max }}{g} X_{f} .
$$

If we now consider water waves propagating in deep water, in the presence of uniform currents, the dispersion Eq. (4) can be modified to take a Doppler shift into account, and now reads

$$
(\omega-k U)^{2}=g k
$$

This specific case was investigated in Touboul et al. [28], where Eq. (5) was demonstrated not to be solution anymore, but to be replaced with

$$
\frac{\partial \omega}{\partial t}=\frac{\frac{d \omega_{0}}{d \tau}}{1+\frac{2 g x}{\left[g+2 U_{0}(\omega-k U 0)\right]^{2}} \frac{d \omega_{0}}{d \tau}}
$$

This is true, since the group velocity admits a new expression, $C_{g}=U_{0}+$ $g /\left(2 \omega-k U_{0}\right)$. Equation (9) shows a difference in the kinematics of the wave group, which becomes more complicated. Indeed, the denominator is now a function of time, and is equal to zero for several values of space and time. The focusing point is not a unique singularity anymore, and the waves present in the group do not merge at a single location of time and space. The singularity is now spread over a focusing area, ranging from $L_{\min }$ and $L_{\max }$, where

$$
L_{\min }=X_{f}\left(1+\frac{2 U_{0} \sigma_{\min }}{g}\right)^{2}, \text { and } L_{\max }=X_{f}\left(1+\frac{2 U_{0} \sigma_{\max }}{g}\right)^{2},
$$

where $\sigma_{\min }$ and $\sigma_{\max }$ are the intrinsic, Doppler shifted, frequencies, respectively given by $\sigma_{\min }=\left(\omega_{\min }-k_{\min } U_{0}\right)=\sqrt{g k_{\min }}$, and $\sigma_{\max }=\left(\omega_{\max }-k_{\max } U_{0}\right)=\sqrt{g k_{\max }}$.

In a recent work, Touboul and Kharif [32] investigated the evolution of a chirped wave packet in the presence of a horizontally constant current presenting linear variations with respect to depth, so that 


$$
U(z)=U_{0}+S z
$$

where $U_{0}$ is the current velocity at the undisturbed free surface, and $S$ is the value of the shear of the current. As an immediate consequence, the vorticity vector associated to this flow field is given by $\boldsymbol{\Omega}=\nabla \wedge \boldsymbol{U}=(0, S, 0)$. In such a configuration, the dispersion equation is also known analytically (see, e.g., $[13,18,29]$ )

$$
\left(\omega-k U_{0}\right)\left(\omega-k U_{0}+S\right)=g|k|
$$

and the related group velocity now reads $C_{g}=U_{0}+g /\left[\sigma_{0}+\sigma_{2}\right]$, where $\sigma_{0}$ and $\sigma_{2}$ are respectively the Doppler shifted intrinsic frequencies $\left(\omega-k U_{0}\right)$ and $\left(\omega-k U_{2}\right)=$ $\left(\omega-k\left(U_{0}-S / k\right)\right)$. In such configuration, neither Eq. (5) nor Eq. (9) is the solution of Eq. (1) anymore. Instead, the solution (3) reads

$$
\frac{\partial \omega}{\partial t}=\frac{\frac{d \omega_{0}}{d t}}{1+\frac{2 g x}{\left[g+U_{0}\left(\sigma_{0}+\sigma_{2}\right)\right]^{2}} \frac{d \omega_{0}}{d t}} .
$$

Here again, the focusing point turns out to be a focusing area, where the values of $L_{\min }$ and $L_{\max }$ are now given by

$$
L_{\text {min }}=X_{f}\left(1+\frac{U_{0}\left(\sigma_{0, \min }+\sigma_{2, \min }\right)}{g}\right)^{2}, \text { and } L_{\max }=X_{f}\left(1+\frac{U_{0}\left(\sigma_{0, \text { max }}+\sigma_{2, \text { max }}\right)}{g}\right)^{2}
$$

where $\sigma_{0, \min }, \sigma_{2, \min }, \sigma_{0, \max }$ and $\sigma_{2, \max }$ are respectively the minimum and maximum of intrinsic frequencies $\sigma_{0}$ and $\sigma_{2}$.

The latter formulation might reduce to both previous cases. Indeed, for $\mathrm{S}=0$, we find the result $\sigma_{0}=\sigma_{2}$, and Eq. (14) reduces to Eq. (10). Furthermore, with $U_{0}=0$, this equation further reduces to $L_{\min }=L_{\max }=X_{f}$, correspondingly to the solution (7) of Eq. (5).

Still, as pointed out in Touboul and Kharif [32], solution (14) also has an interesting behavior, when investigating the only effect of vorticity $S$, independently of any surface current $U_{0}$. Indeed, when considering the absence of surface current, $U_{0}=0$, a nonzero vorticity $S \neq 0$ turns out to change the focusing area in a single point. All frequencies have group velocities affected in such a way that the focusing point is not affected by the value of the vorticity. On the other hand, the focusing time is significantly affected, and all components of the chirped wave packet will reach the focusing point $X_{f}$ at a time $T$ given by

$$
T=T_{f}+\frac{S}{g} X_{f}
$$

This result is very different from what was observed in the presence of uniform current. Indeed, when uniform current was considered, each component was affected 
by Doppler shift, so that the focusing was not optimal. Here, the components are also affected, but the focusing point remains unchanged. This means that the focusing of energy remains optimal, even if delayed or leaded.

These three configurations are interesting, since they admit analytical solutions, allowing to emphasize the effect of a uniform current, or a sheared current, on the focusing behavior of a chirped wave packet. But another effect, of possible great significance, was not considered here. This effect is the influence of the water depth, which has, as it is classically known, a significant impact on dispersive properties of water waves. This idea was raised by Pr. Ezersky, and investigated both numerically and experimentally in Merkoune et al. [21]. In this work, we extend this analysis to the presence of a linearly sheared current.

In the latter case, the above derivation of $C_{g}$ and $\partial C_{g} / \partial \omega$ can still be performed. The dispersion equation now reads $\sigma_{0} \sigma_{2}=g k \tanh (k h)$, where $\sigma_{0}$ and $\sigma_{2}$ are still the previous Doppler shifted frequencies, but now read respectively $\left(\omega-k U_{0}\right)$ and $\left(\omega-k U_{0}+S\right.$ th $\left.(k h)\right)$. This new dispersion equation provides the following expression:

$$
C_{g}=U_{0}+\frac{g \tanh (k h)}{\sigma_{0}+\sigma_{2}}+\frac{g k h+\sigma_{0} S h}{\sigma_{0}+\sigma_{2}}\left(1-\tanh (k h)^{2}\right)
$$

Starting from this group velocity, and after some algebra, one may derive the expression of $\partial C_{g} / \partial \omega$. We obtain

$$
\frac{\partial C_{g}}{\partial \omega}=-2 \frac{\left(C_{g}-U_{0}\right)^{2}}{\left(\sigma_{0}+\sigma_{2}\right) C_{g}}+2 \frac{\left(1-\tanh (k h)^{2}\right)}{\left(\sigma_{0}+\sigma_{2}\right) C_{g}}\left(g h+\left(g k h^{2}+\sigma_{0} S h^{2}\right) \tanh (k h)\right)
$$

Finally, introducing these values in the denominator of Eq. (3) leads to the following relationship:

$$
\left.\left.\frac{x}{C_{g}^{2}} \frac{\partial C_{g}}{\partial \omega} \frac{d \omega_{0}}{d \tau}=-\frac{g x}{X_{f}} \frac{\left(C_{g}+U_{0}\right)^{2}}{\left(\sigma_{0}+\sigma_{2}\right) C_{g}^{3}}+\frac{g x}{X_{f}} \frac{\left(1-\tanh (k h)^{2}\right)}{\left(\sigma_{0}+\sigma_{2}\right) C_{g}^{3}} g h+g k h^{2}+\sigma_{0} S h^{2}\right) \tanh (k h)\right)
$$

Here again, we notice the complexity of Eq. (18), which is time dependent. Indeed, the denominator of Eq. (3) now admits an infinity of singularities, ranging from

$$
\begin{aligned}
& L_{\text {min }}=X_{f} \frac{\left(\sigma_{0, \text { min }}+\sigma_{2, \text { min }}\right) C_{g}^{3}\left(\omega_{\text {min }}\right)}{g} \times F\left(\omega_{\min }, k_{\text {min }}\right), \text { and } \\
& L_{\text {max }}=X_{f} \frac{\left(\sigma_{0, \text { max }}+\sigma_{2, \text { max }}\right) C_{g}^{3}\left(\omega_{\max }\right)}{g} \times F\left(\omega_{\max }, k_{\text {max }}\right),
\end{aligned}
$$

where the function $F(\omega, k)$ is given by

$$
F(\omega, k)=\frac{1}{\left(C_{g}(\omega)+U_{0}\right)^{2}+\left(g h+\left(g k h^{2}+\sigma_{0}(\omega, k) S h^{2}\right) \tanh (k h)\right)\left(1-\tanh (k h)^{2}\right)} .
$$




\section{Results and Discussion}

Results presented here aim to describe the influence of bathymetry, surface current, and vorticity on the focusing behavior of a wave packet. To achieve this goal, the chirped wave packet considered here corresponds to the packet studied in a previous work by Touboul and Kharif [32]. The focusing wave group considered had a frequency varying from $f_{\max }=1.3 \mathrm{~Hz}$ to $f_{\min }=0.7 \mathrm{~Hz}$. The peak frequency is thus given by $\omega_{\text {mean }}=2 \pi\left(f_{\max }+f_{\min }\right) / 2=2 \pi \mathrm{rad} / \mathrm{s}$. This chirped wave packet, when propagating in deep water, in the absence of current and vorticity, presented a focusing location of $X_{f}=16.26 \mathrm{~m}$ and a focusing time of $T_{f}=27 \mathrm{~s}$.

To illustrate the kinematic effects of bathymetry, surface current, and vorticity, we focus in the following subsections on the spreading of the focusing point. Based on the expression of the minimum and maximum distance for caustic formation, $L_{\text {min }}$ and $L_{\max }$, provided by Eq. (19), the spreading of the focusing area can be described as

$$
A_{\text {spread }}=\frac{L_{\max }-L_{\min }}{X_{f}}
$$

This quantity will provide good insights on how vorticity, surface current, and bathymetry affect dispersion.

\section{a. Effect of vorticity and surface current}

In this section, we focus on the combined effects of vorticity and a surface current, homogeneous with depth, on the spreading area $A_{\text {spread }}$. In every case considered, the bathymetry effects are neglected, and water waves propagating in deep water are considered.

Figure 1 depicts the spreading area $A_{\text {spread }}$ of the chirped wave packet as a function of the normalized vorticity $S / \omega_{\text {mean }}$. In the figure, various lines are represented. These lines are colored as a function of the reference current velocity $U_{0} / c_{\text {mean }}$. The word "reference" here means the wavenumber considered that corresponds to the wavenumber computed in the absence of vorticity $\left(S / \omega_{\text {mean }}=0\right)$ for the mean pulsation of the wave packet $\omega_{\text {mean }}$.

This figure shows the strong dependence of the focusing area to both the vorticity and the surface current, in a nontrivial coupling. Indeed, it appears that for strong currents, either positive or negative, the width of the focusing area is sensitive to the vorticity. For positive values of the surface current, the width of the area increases with the vorticity, while it decreases when considering negative values of the surface current. Furthermore, when considering counter flowing currents (negative values of $U_{0} / c_{\text {mean }}$ ), the spreading of the focusing area tends to be limited. This is connected to the limiting value of the blocking current velocity. When considering positive values of the current, this limiting behavior is not observed, and the focusing area seems to be unbounded. One may observe it is greater than 1, meaning the width of the focusing is as wide as the focusing distance, for relatively weak values of the surface current. 


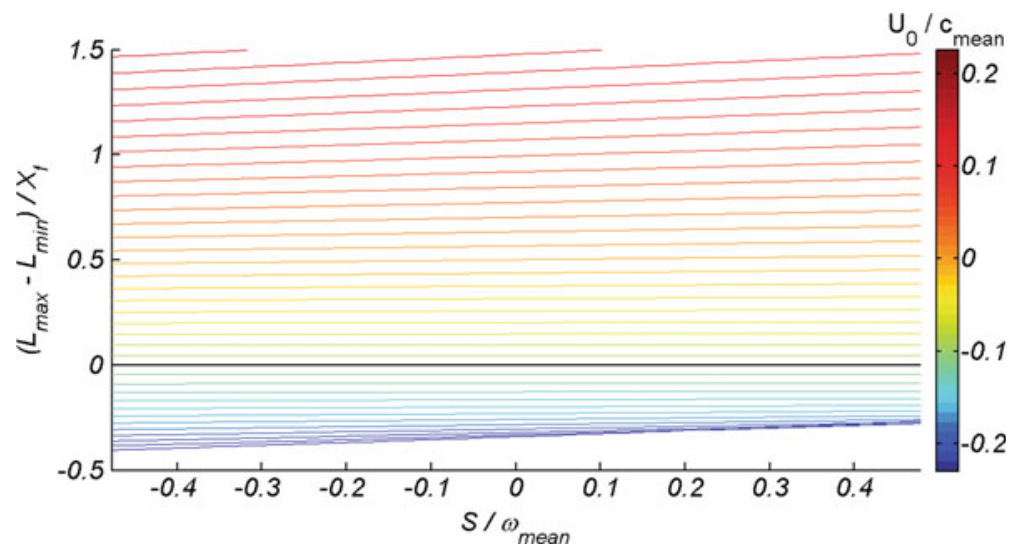

Fig. 1 Spreading area $A_{\text {spread }}$ of the focusing wave packet plotted versus normalized vorticity $S / \omega_{\text {mean }}$. Various lines are colored as a function of the normalized reference current velocity $U_{0} / c_{\text {mean }}$

In the meantime, when considering zero surface current (this case is emphasized by the thick black line in Fig. 1), the focusing area turns out to remain a singularity, corresponding to a focusing point, and to be insensitive to the vorticity. This is a confirmation of the result initially obtained in Touboul and Kharif [32]. This result appears not to remain valid for constant, but nonzero values of the surface current.

The dependence of the width of this focusing area can also be analyzed as a function of the normalized effective surface current velocity. The word "effective" here means the phase velocity considered for normalization is still the phase velocity of the average pulsation of the group, $\omega_{\text {mean }}$, but now accounts for the actual value of vorticity. This behavior is presented in Fig. 2, where the spreading area is plotted versus the normalized effective current velocity, $U_{0} / c_{\text {mean }}$. In this figure, various lines appear, colored as a function of the normalized vorticity, $S / \omega_{\text {mean }}$.

Confirming the previous result, every curve intersects in $A_{\text {spread }}=0$ for the value of surface current $U_{0} / c_{\text {mean }}=0$. But beyond this point, it is interesting to notice that the area of focusing always increases with the velocity of the surface current (in modulus). Though, from these curves, it also appears the vorticity has an influence on this behavior, since for strong positive vorticities, the increase rate is smaller than it is when considering strong negative vorticities.

These results are summarized in Fig. 3, where the evolution of the spreading area (in modulus) of the focusing wave packet is presented in the $\left(U_{0} / c_{\text {mean }}, S / \omega_{\text {mean }}\right)$ plane. From this figure, the dependence of the focusing area to both surface current velocity and vorticity might become more intuitive. It appears that for zero values of the surface current, the focusing area is always zero, corresponding to optimal focusing. But this focusing area is found to depend on both surface velocity and vorticity. However, this coupling is nontrivial, since the enlargement of the focusing area is more sensitive to the surface current when considering strong negative 


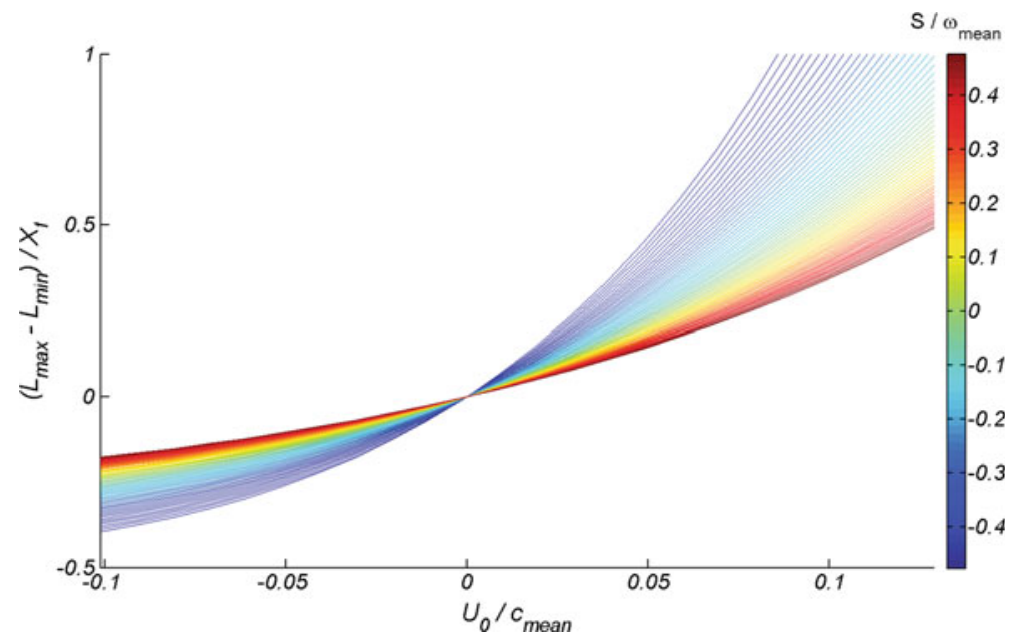

Fig. 2 Spreading area $A_{\text {spread }}$ of the focusing wave packet plotted versus normalized effective current velocity $U_{0} / c_{\text {mean }}$. Various lines are colored as a function of the normalized vorticity $S / \omega_{\text {mean }}$

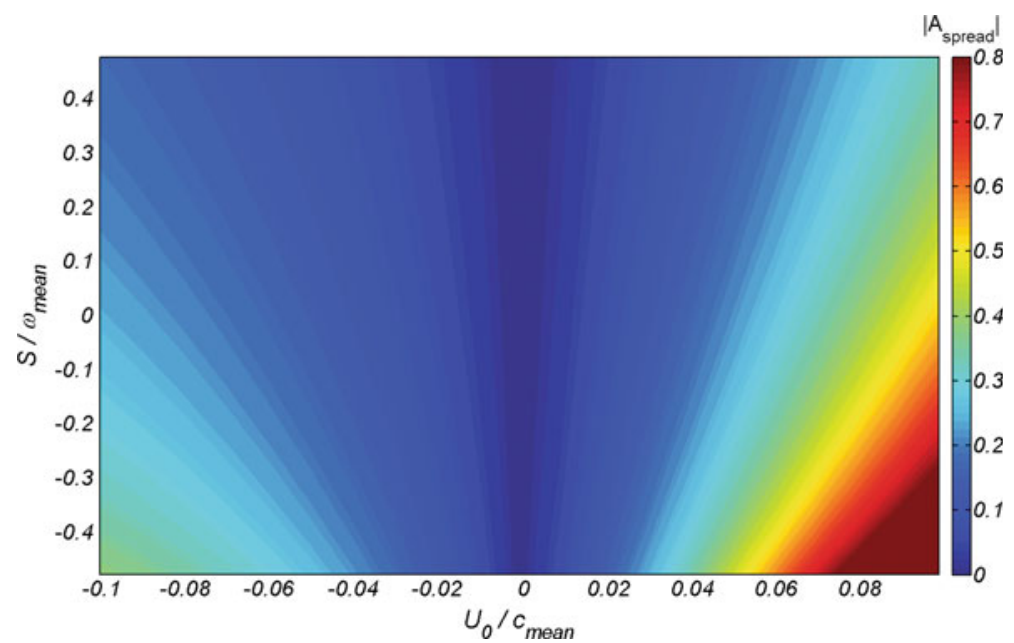

Fig. 3 Evolution of the modulus of the focusing area in the $\left(S / \omega_{\text {mean }}, U_{0} / c_{\text {mean }}\right)$ plane

vorticity. It can also be emphasized that the dependence of this area with vorticity is more important when considering strong values (positive or negative) of the surface velocity.

\section{b. Effect of Vorticity and Bathymetry}

In this section, we focus on the combined effects of bathymetry and vorticity on the spreading area $A_{\text {spread }}$. In every case considered, the surface current velocity is taken equal to zero. 


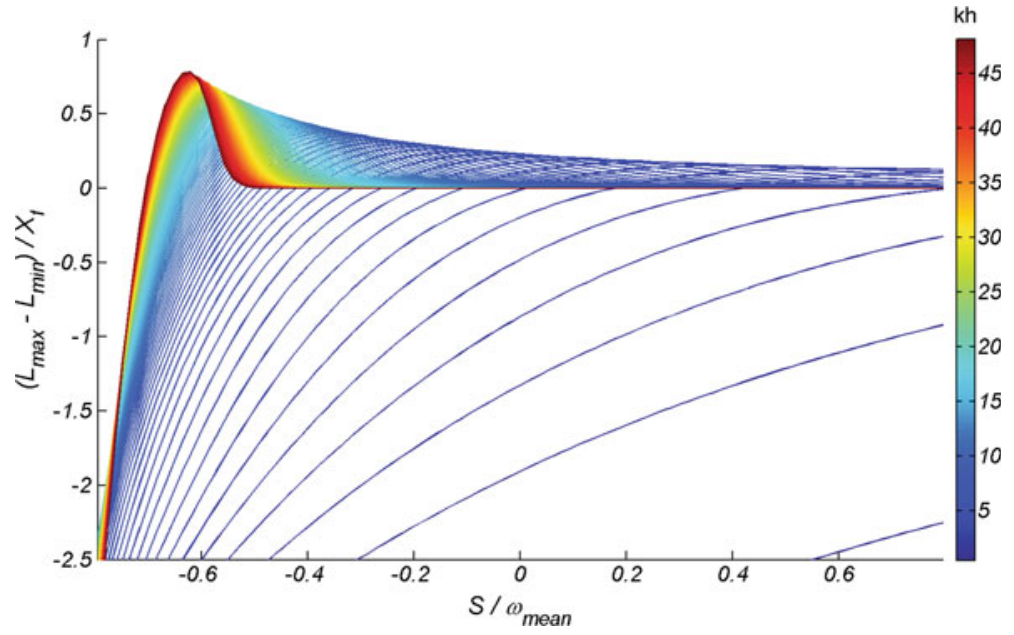

Fig. 4 Spreading area $A_{\text {spread }}$ of the focusing wave packet plotted versus normalized vorticity. Various lines are colored as a function of the reference depth parameter $k h$

Figure 4 depicts the spreading area $A_{\text {spread }}$ of the chirped wave packet as a function of the normalized vorticity $S / \omega_{\text {mean }}$. On the figure, various lines are represented. These lines are colored as a function of the reference depth parameter $k h$. The word "reference" means the wavenumber considered that corresponds to the wavenumber computed in the absence of vorticity $\left(S / \omega_{\text {mean }}=0\right)$ for the mean pulsation of the wave packet $\omega_{\text {mean }}$. From this figure, various behaviors appear for large or small values of $k h$. Indeed, for large values of the depth parameter, the spreading area $A_{\text {spread }}$ is zero for almost every value of the vorticity. This is a confirmation of the result initially obtained in Touboul and Kharif [32] for wave packets propagating in deep water. In this study, the focusing location was found to be unaffected, whatever the value of the vorticity. The vorticity, in deep water, has no effect on the dispersive behavior of the chirped wave packet.

On the other hand, for strong negative values of the vorticity $S / \omega_{\text {mean }}$, Fig. 4 shows a spreading of the focusing area, even for the strongest values of the $k h$ parameter (up to values of $k h=48$ ). These values of the vorticity correspond to a strong coflowing current, which will intuitively result in large values of the wavelength. Thus, the effective wavenumber, taking the influence of the vorticity into account, will be smaller, and the influence of the bathymetry will have a significant effect on the dispersive behavior of the wave packet, resulting in a spreading of the focusing area.

When considering the smallest values of the reference depth parameter $k h$, it turns out that the spreading area is not equal to zero. This means no optimal focusing can be reached in such conditions, and the influence of bathymetry is predominant, regardless to the value of vorticity. Nevertheless, it seems the value of zero will eventually be reached asymptotically, for values of the vorticity large enough. 


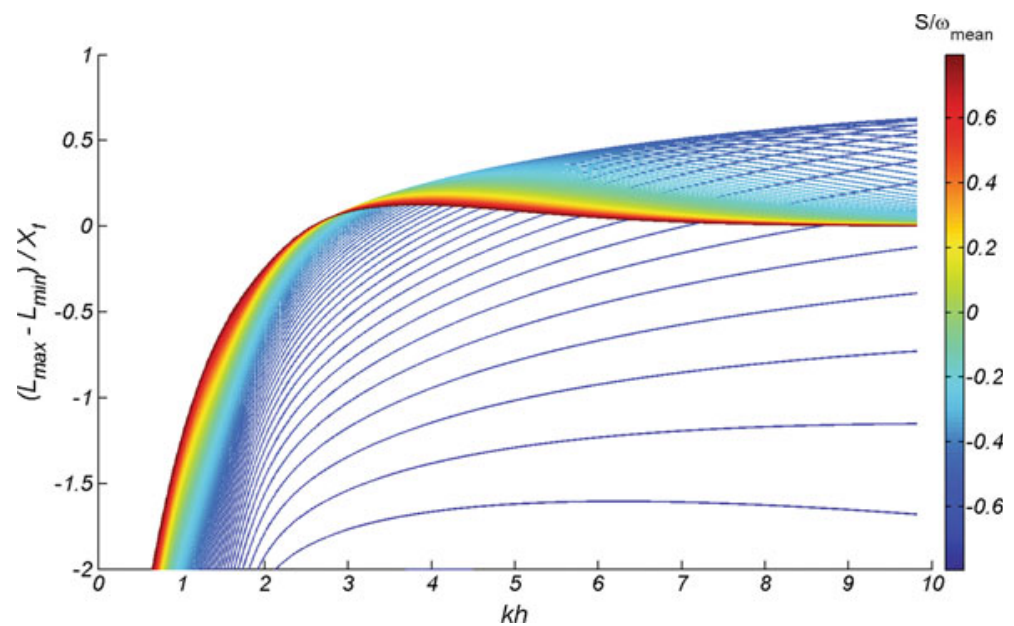

Fig. 5 Spreading area $A_{\text {spread }}$ of the focusing wave packet plotted versus depth parameter $k h$. Various lines are colored as a function of the normalized vorticity $S / \omega_{\text {mean }}$

Given the previous results, it is also interesting to consider the influence of effective depth parameter $k h$ on the focusing area $A_{\text {spread }}$. This is the purpose of Fig. 5, which presents the evolution of the spreading area as a function of the effective depth parameter. The word "effective" here means $k h$ is evaluated using the real wavenumber $\mathrm{k}$, computed using the mean pulsation of the wave packet $\omega_{\text {mean }}$, and taking the real vorticity value into account. In this figure, several lines are observed, colored as a function of the normalized vorticity magnitude, $S / \omega_{\text {mean }}$.

In this figure, we observe various behaviors given the magnitude of the vorticity considered. Indeed, when considering strong vorticity, the spreading area appears to tend to a zero value, meaning the parameter $k h$ has less influence when vorticity is important. On the opposite, for strong, but negative values of the vorticity, the parameter $k h$ is found to have a strong influence on the focusing area of the chirped wave packet.

The values of the parameter $k h$ are striking. Indeed, in the absence of vorticity, depicted by the green lines in Fig. 5, the bathymetry seems to have an influence up to $k h=7$. This is explained through the frequency width involved in the chirped wave packet. But in the meantime, these values are larger than 10 when vorticity becomes important, but negative.

In Fig. 6, the evolution of the spreading area (in modulus) of the focusing wave packet is presented in the $\left(S / \omega_{\text {mean }}, k h\right)$ plane. From this figure, it appears that the focusing area is zero, corresponding to optimal focusing, for deep water conditions, and for positive values of the vorticity. On the other hand, the important influence of the depth parameter on the focusing area appears clearly on that figure, when considering important opposing vorticities. This observation is probably explained through a broadening of the spectral width of the group in the presence of vorticity. 


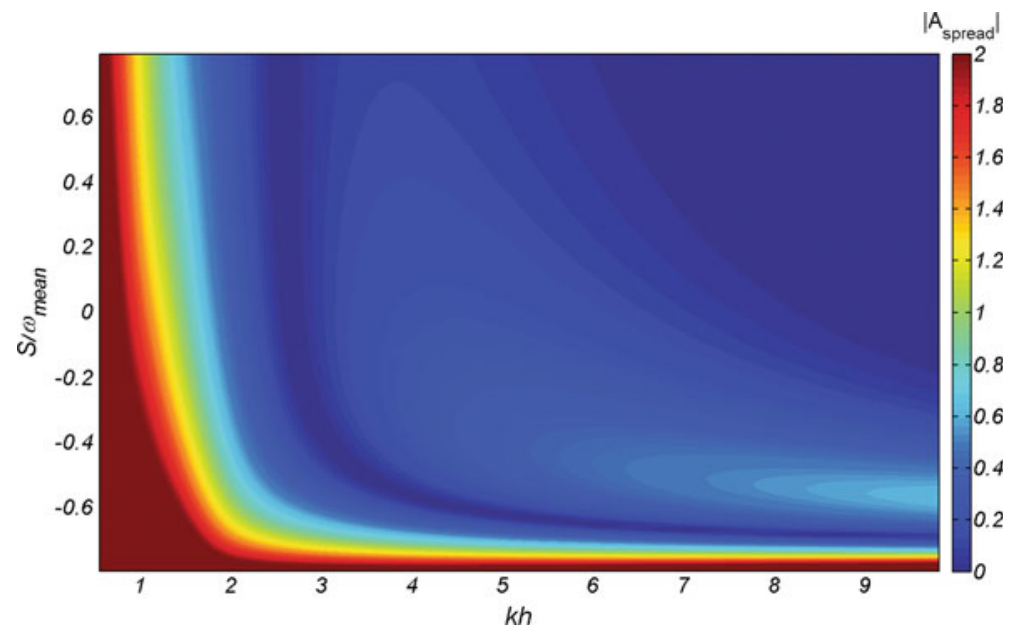

Fig. 6 Evolution of the modulus of the focusing area in the $\left(S / \omega_{\text {mean }}, k h\right)$ plane

Thus, the mean frequency is not accurate enough to provide a good indicator of the depth effects on the spreading area. Indeed, components involved in the wave packet are now sensitive to finite depth effects, resulting in a spreading of the focusing area. Same remark can be done when considering the vorticity effects for waves propagating in shallow water (small values of the $k h$ parameter). When components of the group tend to become nondispersive, due to shallowness, vorticity has no influence anymore, and the focusing cannot be reached anymore.

\section{Conclusion}

The kinematic behavior of a focusing wave group propagating in finite depth, in the presence of constant vorticity and surface currents is studied analytically. Within the framework of the linear theory we use an approximate kinematic model allowing to describe the focusing point, and its transformation to a focusing area.

Confirming previous results by Touboul and Kharif [34], the effect of vorticity in deep water is found to be surprising, and very different from what is expected in the presence of surface current, varying uniformly with depth. Indeed, when the only vorticity is present, the spreading of the focusing area is not expected anymore. On the other hand, when both current and vorticity are present the focusing area of the wave packets exhibits a dependence on the vorticity. Namely, the modulus of the focusing area always increases with the modulus of the vorticity, whatever its sign. On the other hand, this area is always found to depend on the surface current intensity, with or without vorticity. However, the rate of this dependence is found to be sensitive to the value of vorticity considered. 
The effect of bathymetry on the focusing area is also investigated. Here again, results are surprising, since bathymetry turns out to have a strong impact on the focusing area, even for very large values of $k h$. Indeed, this can be explained by a broadening of the wavelength within the wave group. In the presence of strong negative vorticities, the spatial spectral width is increased in such a way the reference wave number might be misleading. Thus, some parts of the components suffer the influence of the bathymetry where we should not expect it. Influence of bathymetry is thus found, indirectly, to have even more significant impact on the dispersive behavior of the chirped wave packet than surface currents do.

Acknowledgements This work is the continuation of a collaboration initiated jointly with Prof. Alexander Ezersky. The authors are also thankful to the editors for organizing such a manuscript. The authors would like to thank the French DGA, who supported this work through the ANR grant ANR-13-ASTR-0007.

\section{References}

1. Belibassakis K. A., Simon B., Touboul J. \& Rey V. (2017) A coupled-mode model for water wave scattering by vertically sheared currents in variable bathymetry regions, Wave Mot. In Press.

2. Benjamin, T. and Feir, J. (1967). The disintegration of wave train on deep water, J. Fluid Mech. vol. 27, 417-430.

3. Brevik I (1979) Higher-order waves propagating on constant vorticity currents in deep water. Coast Eng 2:237-259.

4. Brown MG (2001) Space-time surface gravity wave caustics: structurally stable extreme events. Wave Mot 33:117-143.

5. Brown MG, Jensen A (2001) Experiments on focusing unidirectional water waves. J Geopys Res 106(C8):16917-16928.

6. Clamond, D. and Grue, J. (2002). Interaction between envelope solitons as a model for freak wave formations. Part I: Long time interaction C. R. Mecanique, vol. 330, 575-580.

7. Constantin A (2011) Two-dimensionality of gravity water flows of constant non zero vorticity beneath a surface wave train. Eur J Mech B/Fluids 30:12-16.

8. Dalrymple RA (1974) A finite amplitude wave on linear shear current. J Geophys Res 79(30):4498-4504.

9. Dyachenko, A.I. and Zakharov, V.E. (2005). Modulation instability of Stokes waves - freak wave, JETP Letters, vol. 81, 6, 255-259.

10. Dysthe, K.B. (2001). Modelling a Rogue Wave - Speculations or a realistic possibility? Rogue Waves, Brest, Ed. by M. Olagnon, and G.A. Athanassoulis, 255-264.

11. Johannessen TB, Swan C (2003) On the nonlinear dynamics of wave groups produced by the focusing of surface water waves. Proc R Soc Lond A 459:1021-1052.

12. Johnson RS (1976) On the modulation of water waves on shear flows. Proc R Soc Lond A 347:537-546.

13. Jonsson IG (1990) Wave-current interactions. In: LeMehaute B, Hanes DM (eds) The sea, vol 9. John, New-York, pp 65-120.

14. Kharif C, Pelinovsky E (2003) Physical mechanisms of the rogue wave phenomenon. Eur J Mech B/Fluids 22:603-634.

15. Kharif C, Pelinovsky E, Talipova T (2001) Focusing of nonlinear wave groups in deep water. JETP Lett. 73(4):170-175. 
16. Kharif C, Giovanangeli J-P, Touboul J, Grare L, Pelinovsky E (2008) Influence of wind on extreme wave events: experimental and numerical approaches. J Fluid Mech 594:209-247.

17. Kharif, C. \& Touboul, J. (2010) Under which conditions the Benjamin-Feir instability may spawn an extreme wave event: a fully nonlinear approach, Eur. Phys. J. Special Topics, 185, p. $159-168$.

18. Kirby JT, Chen TM (1989) Surface waves on vertically sheared flows: approximate dispersion relations, J. Geophys. Res., 94(C1):1013-1027.

19. Kishida N, Sobey RJ (1988) Stokes theory for waves on linear shear current. J Eng Mech 114:1317-1334.

20. Lavrenov, I.V. (1998). The wave energy concentration at the Agulhas current of South Africa, Natural Hazards, vol. 17, 117-127.

21. Merkoune D, Touboul J, Abcha N, Mouaz D, Ezersky A (2013) Focusing wave group on a current of finite depth. Nat Hazards Earth Syst Sci 13:2941-2949.

22. Pak OS, Chow KW (2009) Free surface waves on shear currents with non-uniform vorticity : third order solutions. Fluid Dyn Res 41:1-13.

23. Peregrine DH (1976) Interactions of water waves and currents. Adv Appl Mech 16:9-117.

24. Simmen JA (1984) Steady deep-water waves on a linear shear current. PhD thesis, California Institute of Technology, Pasadena, Californie.

25. Simmen JA, Saffman PG (1985) Steady deep-water waves on a linear shear current. Stud Appl Math 73:35-57.

26. Teles Da Silva AF, Peregrine DH (1988) Steep, steady surface waves on water of finite depth with constant vorticity. J Fluid Mech 195:281-302.

27. Touboul, J., Giovanangeli, J.P., Kharif, C. and Pelinovsky, E. (2006). Freak waves under the action of wind: experiments and simulations, European J. Mech. B/Fluids, vol. 25, 662-676.

28. Touboul J, Pelinovsky E, Kharif C (2007) Nonlinear focusing wave groups on current. J. Korean Soc Coast Ocean Eng 19(3):222-227.

29. Touboul J, Charland J, Rey V, Belibassakis K (2016) Extended mild-slope equation for surface waves interacting with a vertically sheared current. Coast Eng 116:77-88.

30. Touboul, J. \& Kharif, C. (2006) On the interaction of wind and extreme gravity waves due to modulational instability, Phys. Fluids, 18, 108103.

31. J. Touboul \& C. Kharif, "Nonlinear evolution of the modulational instability under weak forcing and damping", Nat. Hazards Earth Syst. Sci., 10, p. 2589-2597(2010).

32. Touboul J, Kharif C (2016) Effect of vorticity on the generation of rogue waves due to dispersive focusing, Nat. Haz., 84(2):585-598.

33. Tsao S (1959) Behavior of surface waves on a linearly varying current. Tr Mosk Fiz Tekh Inst Issled Mekh Prikl Math 3:66-84.

34. Whitham GB (1974) Linear and nonlinear waves. Wiley, New York.

35. White, B.S. and Fornberg, B. (1998). On the chance of freak waves at sea, J. Fluid Mech., vol. $355,113-138$. 